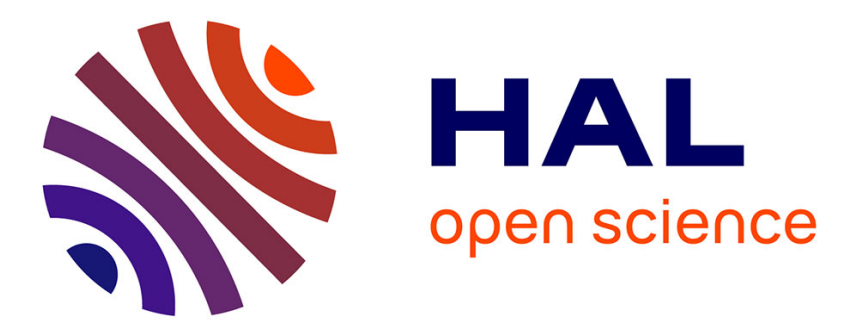

\title{
Réseau, capital social et performance pour l'organisation: le cas des responsables de sites de prestation logistique
}

\author{
Franck Brulhart, Sophie Claye-Puaux
}

\section{- To cite this version:}

Franck Brulhart, Sophie Claye-Puaux. Réseau, capital social et performance pour l'organisation : le cas des responsables de sites de prestation logistique. Revue management \& avenir, 2009, 4 (24), pp.65-82. halshs-00431201

\section{HAL Id: halshs-00431201 \\ https://shs.hal.science/halshs-00431201}

Submitted on 8 Dec 2015

HAL is a multi-disciplinary open access archive for the deposit and dissemination of scientific research documents, whether they are published or not. The documents may come from teaching and research institutions in France or abroad, or from public or private research centers.
L'archive ouverte pluridisciplinaire HAL, est destinée au dépôt et à la diffusion de documents scientifiques de niveau recherche, publiés ou non, émanant des établissements d'enseignement et de recherche français ou étrangers, des laboratoires publics ou privés. 


\title{
Réseau, capital social et performance pour l'organisation : le cas des responsables de sites de prestation logistique
}

\author{
Network, social capital and organization performance: \\ The case of third-party logistics site managers
}

\author{
Franck BRULHART et Sophie CLAYE-PUAUX
}

\begin{abstract}
Franck Brulhart (*)
Franck Brulhart est Maître de Conférences en Sciences de Gestion à l'Université de la Méditerranée (Aix-Marseille II) et membre du LEST - UMR CNRS 6123. Diplômé de l'EDHEC, Agrégé d'économie et gestion et détenteur d'un doctorat en sciences de gestion, il mène actuellement des recherches sur le thème de la coopération interentreprises et des réseaux. Il a notamment publié ses résultats dans M@n@gement, Finance-Contrôle-Stratégie, Management International, Observer pour Comprendre, Revue Française de Gestion Industrielle.
\end{abstract}

franck.brulhart@univ-amu.fr

\section{Sophie Claye-Puaux}

Sophie Claye-Puaux est Maître de Conférences en Sciences de Gestion à l'Université de la Méditerranée (Aix-Marseille II) et membre du CRET-LOG d'Aix-en-Provence. Ses recherches portent sur les dimensions stratégiques, organisationnelles et sociales des supply chains et des organisations logistiques. Elle a notamment publié ses résultats dans Logistique et Management.

sophie.puaux@univ-amu.fr

(*) Auteur correspondant à l'adresse suivante :

Faculté des Sciences Economiques et de Gestion

14 Avenue Jules Ferry

13621 Aix en Provence cedex 


\title{
Réseau, capital social et performance pour l'organisation : le cas des responsables de sites de prestation logistique
}

\author{
par Franck Brulhart et Sophie Claye-Puaux
}

\begin{abstract}
Résumé La structure et la nature du réseau social d'un responsable de site de prestation logistique impactent-elles sa capacité à améliorer la performance de son site? Bâti sur une revue de la littérature dans les domaines de la prestation de services logistiques et de la théorie du capital social et sur une recherche empirique exploratoire, cet article fournit et discute un ensemble de propositions répondant à cette question de recherche.
\end{abstract}

Abstract Does the social network developed by managers of third-party logistics site impact their ability to improve the site's performance? Based on a literature review in the fields of third-party logistics and social capital theory, and on an exploratory study, this article provides and discusses a set of propositions answering that research question.

S'interroger sur les facteurs de performance des sites de prestation logistique n'est pas anodin. $\mathrm{Au}$ sein des supply chains industrielles et commerciales, les prestataires de services logistiques (PSL) jouent en effet un rôle de plus en plus central : garants de la bonne coordination des flux de marchandises et d'information, la performance opérationnelle des PSL conditionne directement celle des supply chains dans lesquelles ils sont intégrés ${ }^{1}$. Or, si le nombre de recherches portant sur les PSL croit régulièrement depuis une quinzaine d'années, peu de recherches ont cherché à ce jour à identifier les facteurs de performance des sites de prestation logistique: les thématiques développées par le passé concernent principalement le processus d'externalisation logistique, ses bénéfices pour le chargeur ou pour la supply chain dans son ensemble, la nature des relations PSL-chargeur en découlant, ou encore l'évolution des métiers de la prestation logistique (Marasco, 2008).

Les études traitant des facteurs de performance des sites logistiques se concentrent sur l'impact des facteurs technologiques (systèmes d'information, automatismes, logiciels) et des facteurs liés aux ressources humaines $(\mathrm{RH})$ sur la performance comprise comme productivité et respect des délais et des niveaux service offerts au client. Pour autant la performance des sites de prestation logistique ne se résume plus aujourd'hui à la performance d'exploitation : de plus en plus, elle s'exprime également en termes de capacité à proposer de nouveaux assemblages de ressources (Fulconis et Paché, 2005). Ainsi la mission des cadres intermédiaires de la prestation logistique, les responsables de sites, relève à la fois de l'optimisation du fonctionnement des sites et de l'innovation dans l'offre proposée (Camman et Livolsi, 2007).

Parallèlement, un consensus s'est désormais établi concernant la valeur attachée au capital social, défini comme l'ensemble des liens sociaux accumulé par les acteurs. Si le débat est toujours ouvert quant aux mécanismes par lesquels le capital social influence la performance, les auteurs structurent de plus en plus systématiquement la réflexion autour de deux formes de performance générées par le capital social : la performance d'exploitation et la performance

\footnotetext{
${ }^{1}$ Voir la contribution dans ce même numéro de François Fulconis, Marlène Monnet et Gilles Paché
} 
dite d'innovation ou de créativité. La théorie du capital social est ainsi singulièrement porteuse de sens pour qui souhaite identifier les facteurs déterminants de la performance comprise sous ces deux angles. En outre, la théorie du capital social permet de faire le lien entre niveau de l'individu, le niveau de l'organisation et le niveau inter-organisationnel.

Partant, nous posons la question de recherche suivante : la structure et la nature du réseau social d'un responsable de site de prestation logistique impactent-elle sa capacité à améliorer la performance (d'exploitation et d'innovation) de son site ? Une revue de littérature sur la prestation de services logistiques (1) couplée à la théorie du capital social nous permettra d'émettre des propositions de recherche (2). Confrontées à une étude exploratoire qualitative, ces propositions seront par la suite discutées (3).

\section{Les PSL au cour de la performance des supply chains}

Les prestataires de services logistiques suscitent depuis quelques années une attention croissante de la part des chercheurs en gestion, en témoigne l'article récent de Marasco (2008) proposant une synthèse de la littérature produite au cours des 20 dernières années sur le sujet dans 33 journaux académiques de réputation internationale. Il ressort de cet article que le sujet des PSL a réellement émergé à partir de 1995, et qu'il a connu un fort développement à partir de 2001 seulement. Ce succès récent dans la sphère académique atteste d'un secteur lui-même jeune et en transformation rapide ; ce succès a en revanche pour l'avenir toutes les chances de perdurer, le phénomène d'externalisation des activités logistiques auprès de prestataires spécialisés croissant toujours régulièrement tout en s'accompagnant d'enjeux opérationnels et stratégiques renforcés (Ashenbaum et al., 2005 ; Lieb et Butner, 2007). Acteur central de la supply chain, le PSL doit développer et consolider une double compétence liée d'une part à sa capacité d'organisation mais aussi à sa capacité d'innovation.

\subsection{Le PSL : acteur stratégique de la chaîne logistique}

Issu donc d'un secteur en constante évolution, le PSL, défini au premier abord comme une entreprise réalisant pour le compte d'un client, le «chargeur», tout ou partie des opérations logistiques, s'inscrit de plus en plus souvent dans les supply chains comme un véritable «gestionnaire d'interfaces » (Fulconis et Paché, 2005). Son métier recouvre une palette d'activités qui s'est considérablement élargie et enrichie au fil des années : ainsi Berglund et al. (1999), Roques et Michrafy (2003), Sauvage (2003) ou Lai (2004) observent une complexification des métiers de la prestation logistique. L'offre de services logistiques des PSL comprend ainsi des prestations basiques traditionnelles (préparation de commandes, stockage/magasinage, gestion des stocks, transport), des services additionnels (service aprèsvente, facturation pour compte client, archivage), voire de nouveaux métiers (installation de site, co-manufacturing, conditionnement, gestion de centre d'appel, co-packing, colisage, technologies de l'information) (Roques et Michrafy, 2003). Les PSL se distinguent ainsi les uns les autres par le contenu de leur offre de service, plus ou moins complexe, intégrant plus ou moins de technologies de l'information, et générant finalement plus ou moins de valeur ajoutée (Lai, 2004). Notons également une tendance de fond du secteur de la prestation logistique à adapter l'offre aux besoins singuliers des clients ; les PSL se distinguent en ceci notamment des entreprises de transport traditionnelles ou des intégrateurs comme DHL ou Fedex qui proposent des offres purement standard (Hertz et Alfredsson, 2003). C'est probablement ce qui explique que nombre d'auteurs affinent leur définition du PSL, prenant en compte la nature de la relation qui les lie à leurs clients, plus coopérative et mutuellement 
bénéfique, plus stratégique, que les transactions au coup par coup typiques de l'achat traditionnel de transport ou d'entreposage (Marasco, 2008).

Forts de ces activités élargies, les PSL s'insèrent dans les chaînes logistiques à une place progressivement stratégique : garants de la coordination de flux physiques, d'information voire financiers parfois complexes, les PSL se retrouvent au cœur de la réalisation du «projet» des supply chains. Par la mise à disposition efficace de moyens techniques et humains et de compétences en gestion d'interfaces, les PSL assurent en effet la gestion des flux tout en visant l'optimisation du triptyque coût / qualité de service / délai (Fulconis et Paché, 2005). Parmi les bénéfices du recours à un PSL les plus régulièrement observés par les chercheurs, on trouve plus précisément : la réduction des coûts logistiques, l'amélioration des niveaux de service, l'amélioration du niveau de satisfaction du consommateur final, l'amélioration de l'exploitation des technologies, la réduction des investissements en infrastructures, équipements et ressources humaines, l'augmentation de la flexibilité et de la productivité, et l'accès à des compétences nouvelles (Marasco, 2008). La performance du PSL génère ainsi non seulement un avantage compétitif pour lui-même, mais également pour son client et plus largement pour la supply chain organisée autour de ces acteurs : le PSL est bien aujourd'hui un maillon à part entière de la supply chain (Fulconis et Paché, 2005). La reconnaissance par les chargeurs du rôle joué par les PSL dans les supply chains a ainsi conduit les chargeurs à se reposer plus largement sur les compétences d'un nombre limité de prestataires appelés de plus en plus souvent à être force de proposition sur la conception des solutions logistiques (Fulconis et Paché, 2005) tout en opérant un contrôle vigilant de leur activité par le biais de la contractualisation et du suivi d'indicateurs de performance (Sauvage, 2004).

\subsection{Le double rôle « entrepreneurial » et « instrumental » du PSL}

Finalement cette description du métier et de la mission du PSL au sein des supply chains fait apparaître une double exigence dans le développement de ses capacités : il s'agit en effet pour le PSL de faire preuve de «capacités d'organisation» pour assurer la bonne conduite des opérations offertes au client mais également de «capacités d'innovation » pour faire face à de nouvelles contraintes ou opportunités par l'élaboration de nouvelles combinaisons de ressources (Camman et Livolsi, 2007). Or dans ce cadre, les directeurs de sites des PSL sont, " compte-tenu de la multi-localisation des unités de production de services, les garants de la mise en acte de la stratégie et donc des acteurs clés dans les transformations organisationnelles que connaissent les PSL depuis une dizaine d'années " (Camman et Livolsi, 2007, p. 44). Responsables des contrats de prestation sur leurs sites et autonomes quant à l'organisation des sites, au choix des équipements ou des méthodes à exploiter, les directeurs de sites sont les pilotes de la performance, dans une optique de centre de profit. Revêtant à la fois un rôle «instrumental » et « entrepreneurial », le directeur de site doit tout à la fois exploiter au mieux les ressources allouées pour servir son ou ses clients et explorer de nouvelles façons de faire pour améliorer la performance (Camman et Livolsi, 2007).

Les capacités du directeur de site chez les PSL sont donc directement sollicitées pour le développement de la performance du site. Si les écarts de performance entre sites «prestés » ne sauraient évidemment s'expliquer uniquement par les caractéristiques des directeurs de sites, la question de l'impact du directeur de site sur la performance de son ou de ses sites semble pleinement justifiée par son rôle même. Cette question pourrait être abordée de multiples façons : on pourrait par exemple s'interroger sur la formation des directeurs de site. Nous faisons ici le choix d'aborder cette question sous l'angle du réseau social développé par 
les directeurs de sites des PSL. Cette approche nous paraît particulièrement pertinente dans la mesure où la socialisation et la communication interpersonnelle sont souvent évoquées comme facteur de performance dans les relations de supply chain, notamment avec les PSL (Knemeyer et Murphy, 2005). De plus la théorie du capital social est une clé d'entrée pertinente pour opérer le lien entre le niveau de l'individu et le niveau de la performance organisationnelle (Moran, 2005).

\section{Réseau social et performance}

Le réseau social (ou capital social) se définit comme l'ensemble des liens sociaux accumulé par les acteurs. Cet article vise précisément à explorer les relations entre le capital social et la performance. Pour cela, nous distinguerons deux dimensions du capital social (Nahapiet et Goshal, 1998) : la dimension relationnelle (quelle est la nature des relations de l'acteur avec ses partenaires) et la dimension structurelle (avec qui l'acteur est en relation). La dimension relationnelle renvoie ici à la faiblesse ou la force des liens alors que la dimension structurelle renvoie au caractère dense ou lâche du réseau.

Le concept de la force des liens a été développé par Granovetter (1983) dans le but de caractériser la nature des relations sociales. Dans cette perspective, considérant l'ensemble des relations directes d'un individu, la force des liens se définit comme une combinaison de l'intensité de la proximité affective, de la fréquence des interactions et du degré de réciprocité de la relation (Granovetter, 1973). Un lien faible se caractérisera ainsi par une relation distante et une absence de proximité affective, n'impliquant que des contacts rares ou occasionnels ; à l'opposé, un lien fort se définira comme une relation étroite animée par des échanges fréquents et réguliers, construite sur des rapports de confiance et sur une proximité et une familiarité élevée.

La notion de densité du réseau renvoie quant à elle à la présence de nombreux liens indirects entre les membres du réseau ${ }^{2}$. Un réseau dense est donc constitué de nombreux liens indirects alors que la faible proportion des liens indirects dans un réseau est à l'origine des trous structurels qui caractérisent un réseau lâche (Burt, 1992).

\subsection{Liens faibles, réseau lâche et capacité créatrice}

L'argument développé par Granovetter (1973) à propos de la «force des liens faibles » repose sur l'idée que les liens faibles sont plus propices à l'émergence d'idées nouvelles et jouent le rôle d'un pont permettant d'accéder à des informations plus pertinentes et plus récentes. Granovetter (1973) défend ici l'idée que les liens faibles permettent de connecter entre eux des individus appartenant à des cercles sociaux dissociés alors que les liens forts ont de fortes chances de rapprocher des individus appartenant aux mêmes cercles sociaux (Brulhart et Claye-Puaux, 2008). Le faible nombre d'interactions antérieures et l'absence de relations personnelles étroites caractéristiques des liens faibles permettent en ce sens de connecter des acteurs différents, souvent issus de parcours, de formations, d'expériences ou d'origines hétérogènes et ayant peu l'habitude de confronter leurs expertises. C'est cette variété qui est à l'origine d'une information riche et non redondante (Perry-Smith, 2006). Le fait d'avoir accès à des informations non redondantes permet alors de faciliter la créativité, définie comme la capacité à générer des idées, des solutions, des produits ou des processus originaux ou

\footnotetext{
${ }^{2}$ Les liens directs renvoient aux liens existant entre un acteur donné et ses différents contacts alors que les liens indirects font référence aux liens existant entre les contacts d'un même acteur (Podolny et Baron, 1997).
} 
renouvelés. De même, lorsque les contacts d'un acteur sont majoritairement issus d'un même cercle social et caractérisés par une forte homogénéité, la forte proximité entre les acteurs qui en découle limite les opportunités de renouvellement ou de remise en question et bride la possibilité d'un apprentissage en double boucle (Argyris et Schon, 1978). Or, cet apprentissage est propice à la création de nouvelles connaissances (Ingham et Mothe, 2003), qu'elles soient relatives aux idées, aux produits ou aux modes d'organisation. A l'opposé, la diversité et l'hétérogénéité des liens existant au sein d'un réseau formé de liens faibles expose les acteurs à des idées neuves, à des perspectives diversifiées, à des informations renouvelées, à des opportunités nouvelles, permettant l'acquisition ou le développement de nouvelles compétences (McEvily et Marcus, 2005).

A la suite de Granovetter (1973), Burt (1992) élargit l'approche de la notion de liens faibles par le biais de sa théorie des trous structurels («structural holes»). En effet, selon Burt (1992) le bénéfice du réseau social découle du caractère lâche du réseau c'est-à-dire de l'absence de liens entre les membres du réseau relationnel d'un acteur (trous structurels). Lorsque les contacts d'un acteur ne sont pas en relation les uns avec les autres, lorsqu'ils n'appartiennent pas aux mêmes cercles sociaux, ils favorisent l'accès à des informations et des ressources nouvelles, originales et non redondantes (Moran, 2005). De ce fait, la diversité et la variété des liens, caractéristiques des réseaux lâches, sont à l'origine d'un avantage informationnel susceptible de dynamiser les capacités d'innovation de l'acteur (Moran, 2005). Par ailleurs, le fait de disposer de nombreux contacts qui ne sont pas connectés les uns aux autres place l'acteur dans un rôle de «broker» qui lui permet de profiter d'un contrôle supérieur sur la diffusion et de l'utilisation de l'information (Brulhart et Puaux, 2008). Ce positionnement le place dans une position de pouvoir identifiée par Crozier et Friedberg (1977) sous le rôle du «marginal sécant ». Ce contrôle accru permet là encore d'améliorer la capacité des acteurs à mener à bien des tâches créatives (Moran, 2005).

Le responsable de site logistique, en tant que cadre intermédiaire, se doit de développer localement de nouvelles idées ou de nouveaux projets. C'est cette capacité créatrice qui lui permet à la fois de faire évoluer les compétences de l'entreprise (Huy, 2001) et d'assurer la survie et le développement de l'organisation en repensant les modes d'organisation et de pilotage des processus et des activités (Camman et Livolsi, 2007). Pour cela, il est nécessaire qu'il parvienne à réaliser une synthèse des informations internes et externes afin de proposer des alternatives stratégiques innovantes (Floyd et Lane, 2000). En ce sens, le directeur de site doit parvenir à développer une capacité réflexive sur l'organisation et le fonctionnement de son centre de profit (Camman et Livolsi, 2007) lui permettant de mener à bien ce rôle « d'entrepreneur » et de conduite du changement. Or, la position qu'occupe le responsable de site logistique le place souvent au centre d'une série de supply chains imbriquées : en relation directe avec l'ensemble de ses collaborateurs, avec différents fournisseurs, avec ses clients voire avec les clients de ses clients.

Notre objectif est précisément d'explorer les relations existant entre la configuration des relations du responsable de site et de ses interlocuteurs d'une part et la performance exprimée en termes d'innovation et de créativité d'autre part. C'est pourquoi, en appliquant les apports de la théorie du réseau social au cas précis du responsable de site, nous nous interrogeons sur le rôle de la nature et de la structure de son réseau social dans sa capacité d'innovation et de recombinaison de ses activités. A la suite de l'ensemble de ces développements, nous formons donc les deux propositions suivantes : 
Proposition 1: L'existence de liens faibles dans le réseau social du responsable de site logistique est propice à une amélioration de la créativité, de la capacité d'innovation et de la performance du site en matière de lancement de nouveaux projets ou de nouvelles approches.

Proposition 2: Le caractère lâche du réseau social du responsable de site logistique est propice à une amélioration de la créativité, de la capacité d'innovation et de la performance $d u$ site en matière de lancement de nouveaux projets ou de nouvelles approches.

\subsection{Liens forts, réseau dense et optimisation de l'exploitation}

Comme nous l'avons souligné précédemment, les liens forts se caractérisent par l'existence d'interactions fréquentes et régulières, le développement de relations personnelles étroites et l'émergence d'un fort degré de confiance entre les acteurs (Nahapiet et Goshal, 1998). Cet « enchâssement relationnel » («relational embededness ») doit impérativement être pris en compte lorsque l'on considère la performance des échanges. Les contacts proches sur le plan relationnel sont prêts à prendre le temps nécessaire et à faire des efforts pour expliquer, détailler ou être attentif à des idées complexes (Moran, 2005). Les liens forts augmentent la motivation des acteurs et leur implication dans l'échange: ils facilitent ainsi l'accès aux ressources et offrent la possibilité de transférer un volume plus important d'informations (Granovetter, 1983 ; Podolny, 2001). En ce sens, cet accès facilité aux ressources et cette implication plus forte des acteurs permet d'améliorer la performance des tâches routinières et d'optimiser l'exploitation des ressources dans le cadre des processus habituels (Moran, 2005 ; Krause et al., 2007).

Par ailleurs, la notion de liens forts est indissociable du concept de confiance. Celle-ci est en effet dynamisée par l'existence de liens étroits ou d'une proximité affective forte entre les membres du réseau ; de même, la confiance se renforce avec la répétition des interactions et des échanges lorsqu'ils sont fructueux. En entrainant l'assurance que les difficultés intervenant à court terme seront résolues à moyen terme ou qu'un processus d'adaptation permanent se fera de manière mutuellement bénéficiaire (Mohr et Spekman, 1994 ; Dyer et Chu, 2003), la confiance encourage les échanges d'informations et facilite la résolution conjointe des problèmes (McEvily et Marcus, 2005). La confiance associée au développement de liens forts entre les acteurs facilite l'investissement dans les compétences et les routines spécifiques à la relation (Levinthal et Fichman, 1988) et dynamise l'accumulation et le transfert de connaissances (McEvily et Marcus, 2005 ; Krause et al., 2007). La confiance apparaît ici non seulement comme un élément central de la construction du capital social d'un acteur en facilitant l'accès aux autres acteurs (Moran, 2005) mais également comme un atout fondamental dans l'efficacité de la réalisation et la concrétisation des actions mises en œuvre.

Face à la théorie des trous structurels, Coleman $(1988,1990)$ présente une thèse concurrente en avançant l'idée que la force du capital social naît au contraire de l'existence d'un réseau dense formé de relations personnelles étroites et de la multiplication des liens indirects au sein du réseau (Brulhart et Puaux, 2008). Dans cette optique, les réseaux denses permettent, du fait même de la redondance des liens et des informations, de stabiliser des normes et de règles de comportements au sein du réseau, et de favoriser la coopération (Moran, 2005). La cohésion sociale qui naît de ces réseaux denses fait émerger des structures normatives qui guident les comportements (Granovetter, 1992) et offre la stabilité nécessaire à l'exploitation des ressources disponibles au sein du réseau. Les formes d'apprentissage en simple boucle (Argyris et Schon, 1978) sont facilitées et dynamisées. De ce fait la densité du réseau constitue un atout dans la réalisation de procédures routinières ou dans l'exploitation de 
ressources ou de processus existant. Enfin, la proximité forte (qu'elle soit culturelle ou organisationnelle) entre tous les membres d'un réseau dense facilite l'évaluation, la communication et la coordination (Chung et al., 2000 ; Kale et al., 2002 ; Brulhart, 2005) et constitue ainsi un gisement d'efficacité et de performance.

Outre le rôle «d'entrepreneur» du responsable de site évoqué précédemment, celui-ci a également un rôle «instrumental» (Camman et Livolsi, 2007) visant à assurer une exploitation optimale des ressources. Dans ce cadre, sa responsabilité consiste à respecter un cahier des charges déterminé avec le client tout en optimisant le triptyque coût-qualité-délai. Pour cela, le directeur de site logistique doit assurer la mise en œuvre des activités (structuration des processus, coordination des intervenants, mobilisation des technologies et des systèmes d'information) ainsi que le contrôle de ces activités (contrôle ex ante par le biais du respect des procédures et contrôle ex post par le biais du reporting de la performance). La réussite de ces tâches de pilotage conditionne la performance opérationnelle du site et nécessite que le responsable de site développe une forte «agilité opérationnelle » (Christopher, 2000). Or, cette agilité opérationnelle repose en grande partie sur la qualité des interfaces interpersonnelles de la chaîne logistique (Fabbe-Costes, 2005).

C'est pourquoi, nous formons les deux propositions suivantes, visant à éclairer le rôle de la nature et de la structure du réseau social du responsable de site logistique dans sa capacité à optimiser l'exploitation des activités :

Proposition 3: L'existence de liens forts dans le réseau social du responsable de site logistique est propice à une amélioration de la performance du site en matière d'optimisation de l'exploitation des ressources et de réalisation des tâches routinières.

Proposition 4: Le caractère dense du réseau social du responsable de site logistique est propice à une amélioration de la performance du site en matière d'optimisation de l'exploitation des ressources et de réalisation des tâches routinières.

\section{Une exploration de la nature du réseau social et de son impact perçu sur la performance}

\subsection{Méthodologie de la recherche exploratoire}

Nous avons bâti une méthodologie de recherche exploratoire dont l'objectif est de récolter un matériau permettant de discuter les propositions énoncées dans la section 2. Pour cela nous avons réalisé huit entretiens semi-directifs avec des responsables de sites de prestation logistique, visant principalement la description de leurs contacts inter-personnels dans le cadre professionnel. L'analyse de la littérature nous a permis d'établir un plan d'entretien structuré autour de cinq thèmes principaux: identification des interlocuteurs, nature de la relation, contenu des échanges, apports pour le responsable de site, exemples concrets).

Les responsables de sites interrogés sont tous issus d'entreprises de prestation différentes. Ils ont été caractérisées selon leur taille (PME / groupes «familiaux » / grands prestataires internationaux) et selon l'étendue de leur métier (voir section 1) en distinguant ici les prestataires «de services logistiques traditionnels»dont certains ont conservé une forte dominante «transports» des prestataires «de services logistiques à valeur ajoutée ». Les responsables pilotent pour certains un site unique, pour d'autres plusieurs sites; ces sites sont dédiés à un client unique ou multi-clients. La combinaison de ces différents critères nous 
permet d'obtenir un échantillon théoriquement représentatif de responsables de sites de prestation logistique.

Les données collectées lors des entretiens ont été exploitées par analyse thématique (Miles et Huberman, 1994). Nous avons notamment cherché à mettre en évidence les liens forts et faibles des responsables de site, et le caractère plus ou moins dense ou lâche du réseau. Les liens ont été interprétés comme forts lorsque le responsable de site évoquait des échanges fréquents et réguliers, un niveau de confiance élevé, une relation plus étroite avec le contact ; dans le cas contraire le lien a été interprété comme faible. Nous avons considéré que le réseau était dense si le responsable de site estimait que ses contacts se connaissaient et échangeaient eux-mêmes entre eux; dans le cas contraire le réseau était considéré comme lâche. La distinction sur la performance générée par le réseau entre performance de type « exploitation » et de type «créativité » relève également de notre interprétation, fondée sur les termes généraux par lesquels les responsables de sites ont exprimé les apports de leurs échanges et sur les exemples précis d'output évoqués pour les sites.

Le Tableau 1 page suivante présente une synthèse descriptive des données collectées à travers les huit entretiens ${ }^{3}$. Les liens faibles y sont sensiblement moins évoqués, ce qui traduit un biais lié à la méthode de collecte des données, qui a naturellement conduit les répondants à évoquer avant tout leurs contacts "privilégiés »; cependant le biais méthodologique seul n'explique pas ce déséquilibre entre liens forts et liens faibles, nous y reviendrons en conclusion.

\subsection{Discussion des propositions}

Les propositions 1 et 3 articulaient force / faiblesse des liens et capacité d'exploitation / de créativité. Les entretiens conduits sont de nature à illustrer ce premier groupe de propositions. Ainsi les responsables de sites ont évoqué des bénéfices tirés de leurs liens forts que nous avons interprétés en termes d'amélioration de la capacité d'exploitation des sites. Ces liens forts sont noués :

- En interne, avec des collègues responsables de sites ou des collaborateurs, qu'ils soient en relation hiérarchique ou affectés à des fonctions support (service juridique, RH, SI, etc.) ;

- En externe, avec des responsables de sites de prestation logistique concurrents ;

- En externe, dans le cadre de relations d'affaires, avec leurs interlocuteurs clients (il s'agit généralement des responsables logistiques des entreprises clientes) ou fournisseurs (agences d'intérim, fournisseurs d'équipements, sous-traitants transports, agents immobiliers).

\footnotetext{
${ }^{3}$ Note sur la lecture du tableau : les répondants ont identifié des liens inter-personnels ; nous choisissons ici parfois d'y faire référence sous la forme de liens inter-organisationnels (aux clients, fournisseurs, etc.) afin de simplifier la lecture en mettant uniquement en évidence le rattachement organisationnel des contacts identifiés. Par ailleurs, le terme « collègue » désigne un autre responsable de site au sein de la même entreprise ; le terme « collaborateur »désigne un salarié de fonction différente au sein de la même entreprise ; le terme «confrère » désigne un autre responsable de site au sein d'une entreprise concurrente.
} 
Tableau 1 : Une lecture synthétique des données collectées

\begin{tabular}{|c|c|c|c|c|}
\hline Ent & $\begin{array}{c}\text { Profil PSL \& } \\
\text { sites pilotés }\end{array}$ & Liens forts & Liens faibles & $\begin{array}{l}\text { Réseau dense / } \\
\text { réseau lâche }\end{array}$ \\
\hline 1 & $\begin{array}{l}\text { - Groupe international } \\
\text { - Métier : services } \\
\text { logistiques } \\
\text { traditionnels } \\
\text { - Sites dédiés et site } \\
\text { multi-clients }\end{array}$ & $\begin{array}{l}\text { - Avec des collègues } \rightarrow \text { exploitation (RH } \\
\text { disponible, conseils techniques, } \\
\text { informations sur le fonctionnement interne } \\
\text { du groupe) } \\
\text { - Avec des confrères (si relation antérieure } \\
\text { amicale) } \rightarrow \text { exploitation (RH disponible, } \\
\text { évaluations de fournisseurs) } \\
\text { - Avec agences d'intérim } \rightarrow \text { exploitation } \\
\text { (réactivité à nos besoins) } \\
\text { - Avec les clients } \rightarrow \text { exploitation } \\
\text { (benchmarking) }\end{array}$ & $\begin{array}{l}- \text { Avec des collègues } \rightarrow \\
\text { exploitation (conseils techniques) } \\
\text { et créativité } \\
\text { méthodes) }\end{array}$ & $\begin{array}{l}\text { - Réseau interne dense } \\
\text { - Réseau lâche autour des clients } \\
\text { et fournisseurs } \rightarrow \text { créativité } \\
\text { (sources d'idées sur des } \\
\text { techniques différentes issues de } \\
\text { secteurs variés) } \\
\text { - Réseau lâche autour du service } \\
\text { ingénierie interne } \rightarrow \text { créativité } \\
\text { (veille technologique et } \\
\text { organisation) }\end{array}$ \\
\hline 2 & $\begin{array}{l}\text { - Groupe familial (1000 } \\
\text { salariés) } \\
\text { - Métier : services } \\
\text { logistiques à } \\
\text { dominante transports } \\
\text { - Sites multi-clients }\end{array}$ & $\begin{array}{l}\text { - Avec des collaborateurs et collègues } \rightarrow \\
\text { exploitation (échanges et conseils sur des } \\
\text { problèmes sociaux) } \\
\text { - Avec des clients } \rightarrow \text { exploitation } \\
\text { (amélioration du fonctionnement) }\end{array}$ & & $\begin{array}{l}\text { - Réseau interne dense } \\
\text { - Réseau dense au sein des } \\
\text { organisations } \\
\text { professionnelles } \rightarrow \text { syndicales } \\
\text { (informations techniques et } \\
\begin{array}{l}\text { réglementaires, orientations } \\
\text { politiques) }\end{array}\end{array}$ \\
\hline 3 & $\begin{array}{l}\text { - Filiale de groupe } \\
\text { (1200 salariés) } \\
\text { - Métier : services } \\
\text { logistiques } \\
\text { traditionnels } \\
\text { - Site dédié }\end{array}$ & $\begin{array}{l}\text { - Avec d'anciens collaborateurs et } \\
\text { collègues } \rightarrow \text { exploitation (conseils RH, } \\
\text { conseils techniques) } \\
\text { - Avec les collaborateurs } \rightarrow \text { exploitation } \\
\text { (fonctionnement interne) } \\
\text { - Avec le client et les clients du client } \rightarrow \\
\text { exploitation (confiance, fonctionnement et } \\
\text { coordination externe) }\end{array}$ & $\begin{array}{l}\text { - Avec des fournisseurs d'équi- } \\
\text { pements et de NTIC } \rightarrow \\
\text { créativité (évolution des SI, des } \\
\text { matériels) }\end{array}$ & • Réseau interne dense \\
\hline 4 & $\begin{array}{l}\text { - Groupe international } \\
\text { - Métier : services } \\
\text { logistiques à } \\
\text { dominante transports } \\
\text { - Sites multi-clients }\end{array}$ & $\begin{array}{l}\text { - Avec des collègues actuels ou d'anciens } \\
\text { collègues } \rightarrow \text { exploitation (conseils } \\
\text { techniques) } \\
\text { - Avec des collaborateurs } \rightarrow \text { exploitation } \\
\text { (échanges et conseils en lien avec leur } \\
\text { spécialité : RH, SI, juridique...) }\end{array}$ & & $\begin{array}{l}\text { - Réseau dense au sein des } \\
\text { organisations } \\
\text { pyndicales } \\
\text { professionnelles et réunions de } \\
\text { zone } \rightarrow \text { exploitation (partage } \\
\text { d'expériences techniques et RH) } \\
\text { - Réseau lâche autour des clients } \\
\rightarrow \text { créativité (transfert de } \\
\text { pratiques innovantes) }\end{array}$ \\
\hline 5 & $\begin{array}{l}\text { - PME indépendante } \\
\text { ( } 20 \text { salariés) } \\
\text { - Métier : services } \\
\text { logistiques } \\
\text { traditionnels } \\
\text { - Site multi-clients }\end{array}$ & $\begin{array}{l}\text { - Avec les confrères si relation amicale } \rightarrow \\
\text { exploitation (réorienter des contrats) } \\
\text { - Avec les clients } \rightarrow \text { exploitation (aide au } \\
\text { progrès, savoir-faire) } \\
\text { - Avec les collaborateurs } \rightarrow \text { exploitation } \\
\text { (mise en œuvre des projets remportés) }\end{array}$ & $\begin{array}{l}\text { - Avec les confrères à travers les } \\
\text { salons professionnels } \rightarrow \\
\text { créativité (découvrir de nouvelles } \\
\text { compétences et modes } \\
\text { d'organisation) } \\
\text { - Via des réseaux formels profes- } \\
\text { sionnels } \rightarrow \text { créativité (nouvelles } \\
\text { idées sur le plan social) }\end{array}$ & $\begin{array}{l}\text { - Réseau lâche autour des clients } \\
\text { et fournisseurs } \rightarrow \text { créativité } \\
\text { (accéder à de nouvelles } \\
\text { ressources et techniques) }\end{array}$ \\
\hline 6 & $\begin{array}{l}\text { - Groupe international } \\
\text { - Métier : services } \\
\text { logistiques à valeur } \\
\text { ajoutée } \\
\text { - Site dédié et sites } \\
\text { multi-clients }\end{array}$ & $\begin{array}{l}\text { - Avec les clients } \rightarrow \text { exploitation (retour } \\
\text { commercial) } \\
\text { - Avec les agences d'intérim, fournisseurs } \\
\text { d'équipement, les agents immobiliers } \rightarrow \\
\text { exploitation (notamment perf. RH, } \\
\text { réactivité sur nos besoins) } \\
\text { - Avec un confrère (relation amicale) } \rightarrow \\
\text { exploitation (partenaire sur un projet } \\
\text { foncier, échanges sur le métier et les } \\
\text { techniques) } \\
\text { - Avec les collaborateurs et collègues } \rightarrow \\
\text { exploitation (amélioration du management, } \\
\text { des façons de faire, des techniques) }\end{array}$ & $\begin{array}{l}\text { - Via des réseaux formels profes- } \\
\text { sionnels, si convergence des } \\
\text { centres d'intérêt } \rightarrow \text { créativité } \\
\text { (identifier de } \\
\text { opportunités) } \\
\text { - Avec des collègues } \rightarrow \text { créativité } \\
\text { (développement d'activités } \\
\text { nouvelles) }\end{array}$ & $\begin{array}{l}\text { - Réseau interne dense } \\
\text { - Réseau lâche autour du service } \\
\text { ingénierie interne } \rightarrow \text { créativité } \\
\text { (veille technologique, veille } \\
\text { métiers) }\end{array}$ \\
\hline 7 & $\begin{array}{l}\text { - Groupe familial (1000 } \\
\text { salariés) } \\
\text { - Métier : services } \\
\text { logistiques à valeur } \\
\text { ajoutée } \\
\text { - Site dédié }\end{array}$ & $\begin{array}{l}\text { - Avec le client } \rightarrow \text { exploitation (prise en } \\
\text { compte des contraintes, confiance sur le } \\
\text { prix) } \\
\text { - Avec les agences d'intérim, fournisseurs } \\
\text { d'équipement, sous-traitants transport } \\
\rightarrow \text { exploitation (réactivité sur nos besoins, } \\
\text { remontée d'information) } \\
\text { - Avec les confrères sur site (même } \\
\text { immeuble) } \rightarrow \text { exploitation (échanges de } \\
\text { flux) }\end{array}$ & & $\begin{array}{l}\text { - Accès à un réseau lâche via un } \\
\text { collaborateur (de formation, } \\
\text { expérience professionnelle, } \\
\text { réseau professionnel différents } \\
\text { de ceux du répondant) } \rightarrow \\
\text { créativité (proposer de nouvelles } \\
\text { idées au client, de nouvelles } \\
\text { façons de faire) }\end{array}$ \\
\hline 8 & $\begin{array}{l}\text { - Groupe international } \\
\text { - Métier : services } \\
\text { logistiques à valeur } \\
\text { ajoutée } \\
\text { - Site multi-clients }\end{array}$ & $\begin{array}{l}\text { - Avec un client } \rightarrow \text { exploitation } \\
\text { (arrangements mutuels sur problèmes RH) } \\
\text { - Avec agences d'intérim, sous-traitants } \\
\text { transport } \rightarrow \text { exploitation (flexibilité) } \\
\text { - Avec un confrère (relation amicale) } \rightarrow \\
\text { exploitation (échanges \& conseils sur } \\
\text { questions techniques et sociales, } \\
\text { réorientation de clients) } \\
\text { - Avec des collègues } \rightarrow \text { exploitation } \\
\text { (meilleure compréhension des processus) }\end{array}$ & & $\begin{array}{l}\text { - Réseau interne dense } \\
\text { - Accès à un réseau lâche via un } \\
\text { membre de sa famille (métier \& } \\
\text { cercle social complètement } \\
\text { différent) } \rightarrow \quad \underline{\text { créativité }} \\
\text { (nouvelles idées pour sortir de } \\
\text { gros problèmes RH, ex. salariés } \\
\text { dangereux) } \\
\text { - Réseau lâche autour du service } \\
\text { ingénierie interne } \rightarrow \underline{\text { créativité }} \\
\text { (veille technologique, veille } \\
\text { métiers) }\end{array}$ \\
\hline
\end{tabular}


Les responsables de sites ont indiqué que leurs échanges avec ces liens forts leurs permettaient de bénéficier de conseils techniques (conseils juridiques, exploitation des technologies, etc.), d'appuis pour la gestion des ressources humaines et le management (notamment, de «prêt» de main d'œuvre disponible), d'opportunités commerciales (nouveaux contrats, lissage de l'activité commerciale), et de l'amélioration des interfaces externes (meilleures coordination, qualité de service et réactivé avec les fournisseurs ou les clients). L'encadré 1 permet d'illustrer ce propos (Les répondants sont codés R1 à R8 en fonction de leur entreprise d'appartenance).

\section{Encadré 1 - Verbatim : liens forts et performance d'exploitation}

"Je suis très proche de trois autres responsables de sites du groupe, on s'appelle tous les jours ou presque. Ca nous permet d'échanger sur les notes de service du groupe et d'améliorer notre compréhension du process, et puis on s'épaule, on se donne des conseils sur du management par exemple ou sur des questions techniques. » (R8)

"Un sujet d'échange et d'entraide que j'ai souvent avec les autres responsables de site, chez nous ou chez des confrères, c'est sur le recrutement de main d'œuvre. » (R3)

"Avec tous nos fournisseurs, que ce soient les agences d'intérim, les fournisseurs d'équipements, les sous-traitants transport, on a besoin de réactivité et parfois de conseils techniques le plus vite possible : là c'est sûr, avoir une relation personnelle directe forte chez eux nous aide à obtenir cette réactivité, c'est un vrai atout. » (R1)

"Le fait d'avoir eu de bons relationnels et de bonnes expériences avec des interlocuteurs clients ou des anciens collaborateurs nous permet de capitaliser sur ces relations y compris quand ces personnes changent d'entreprise ; il y a souvent un retour commercial. »(R6)

"Le lien très fort avec nos correspondants dans les agences d'intérim est primordial; la masse salariale, c'est le poste de charge le plus important dans nos métiers. » (R6)

"Plus on a une bonne relation avec notre homologue client, plus on a instauré de la confiance, plus on aura de la souplesse pour trouver des solutions aux problèmes qu'on peut rencontrer, et travailler sur des procédures dégradées en cas de besoin par exemple, et en même temps plus on aura d'autonomie pour aménager les process et améliorer l'efficacité du service. On déjeune ensemble très régulièrement, c'est vrai que la relation est conviviale et que ça permet ces échanges. »(R7)

"Moi j'entretiens une relation humaine très étroite avec mes fournisseurs de matériel de manutention, avec les boîtes d'intérim et avec mes sous-traitants transport. C'est grâce à cette relation humaine qu'on est beaucoup plus flexible; avec les sous-traitants transport, le service est même meilleur qu'avec nos propres chauffeurs: eux vont jusque dans les magasins [de la grande distribution] pour récupérer les meubles, et ils nous informent en permanence sur ce qui se passe. »(R7)

"Nos gros clients organisent des échanges réguliers, du suivi avec reporting, actions correctives et autres pour améliorer le fonctionnement. Mais au-delà de la procédure, il y a une dimension relationnelle forte: si on est bien avec eux personnellement, c'est toujours plus productif. » $(R 2)$

"Quand on s'entend bien avec un confrère concurrent, on se rencontre pour voir si on peut s'échanger certains flux ou pour optimiser les flux, par exemple si on a des camions à moitié vides. » $(R 7)$

Il ressort que les liens forts se forment essentiellement selon deux modes : dans les relations d'affaires actuelles (avec les clients ou fournisseurs) d'une part, les responsables de sites perçoivent le lien fort comme une source d'amélioration des performances et cherchent ainsi de manière assez proactive à renforcer les relations avec leurs interlocuteurs directs. Dans 
leurs expériences professionnelles passées d'autre part, les responsables de sites ont identifié des individus (anciens collègues, collaborateurs mais aussi contacts clients ou fournisseurs) possédant une compétence particulière et ont vécu avec eux des expériences professionnelles réussies ayant suscité leur confiance. Ce mode «émergent» de formation des liens forts explique par exemple que les responsables de sites évoquent des liens forts avec des confrères de prestataires concurrents: il s'agit dans de nombreux cas d'anciens collègues ou collaborateurs. Nous remarquons également que les liens amicaux préexistants à l'expérience professionnelle commune ont un rôle mineur alors que les liens amicaux issus de l'expérience professionnelle commune réussie ont un rôle majeur pour les responsables de sites. Autrement dit, les liens amicaux initiés par une relation professionnelle profitable encouragent et dynamisent cette relation alors que les répondants sont plus méfiants quant à la mobilisation de liens purement amicaux dans un contexte professionnel. L'encadré 2 illustre ces différents points.

\section{Encadré 2 - Verbatim : mode de formation des liens forts}

"Le client de mon site a posé des problèmes pendant des années, il a usé du monde ici, à peu près 1300 personnes ont défilé sur ce site de 50 postes. Pour moi, quand je suis arrivé à la direction du site, parvenir à quelque chose cela voulait dire avant tout renouer un lien de confiance avec lui ; j'ai organisé des rencontres plus fréquentes avec mon homologue je lui ai montré qu'on pouvait être au rendez-vous sur leurs attentes, j'ai calmé le jeu. Et puis avec les franchisés [points de livraison clients du chargeur] aussi j'ai tissé des relations directes, les gens nous connaissent et sont en confiance. »(R3)

"Les contacts les plus réguliers et les plus importants pour moi, sont ceux que j'ai avec les autres directeurs de site, que j'ai connus au long de mon cursus, ici et chez mes précédents employeurs. Je suis assez proche d'eux pour plaisanter sur le métier ou d'autres choses mais aussi professionnellement on s'entraide, par exemple quand on recherche du personnel disponible, ou bien quand on veut avoir un avis sur un sous-traitant. » (RI)

"Le réseau se construit essentiellement à travers l'expérience professionnelle, les gens avec qui on a travaillé par le passé ; ça nous permet d'identifier des compétences dans différents domaines, de savoir si on peut faire confiance ; c'est grâce à ce réseau qu'on peut solliciter des expertises au fur et à mesure des besoins. »(R6)

"On bâtit des liens forts sur la compétence et la confiance dans l'efficacité professionnelle, le côté amical des relations est toujours secondaire; il m'est arrivé une fois de travailler avec un fournisseur parce que j'étais proche de lui amicalement et au final ses contreperformances ont mené à l'échec de la relation d'affaires. »(R4)

Les responsables de sites évoquent également des liens plus faibles, qui sont noués en interne ou en externe avec les mêmes types d'acteurs que ceux désignés pour les liens forts. Les bénéfices tirés des liens faibles ont largement été interprétés en termes d'amélioration de la créativité des responsables de sites: sur des thématiques similaires à celles évoquées concernant les liens forts (problèmes techniques, organisation de l'activité, gestion des ressources humaines et management), mais aussi sur la conception de l'offre commerciale (nature du service rendu au client, éventail d'activités proposé), les liens faibles apportent des idées neuves, de l'innovation. Le mode de formation de ces liens faibles relève d'une forme d'institutionnalisation, de «mise en réseau » formalisée, soit en interne par l'entreprise qui incite ses responsables de sites au partage d'expérience en convoquant des réunions de direction annuelles ou bisannuelles, soit en externe par la profession au sens large, à travers les salons ou autres événements associatifs professionnels qui permettent des échanges ponctuels entre confrères notamment. L'encadré 3 illustre ces divers éléments. 


\section{Encadré 3 - Verbatim : liens faibles et créativité}

«Ce n'est pas forcément les gens avec qui on a les contacts les plus fréquents ou les relations de confiance qui nous apportent le plus de choses, parce qu'ils ont une approche peu novatrice ; l'innovation, il faut aller la chercher chez des éléments extérieurs qui peuvent proposer des angles différents. » (R5)

"Les salons nous permettent d'avoir de nouvelles idées, parce qu'on regarde les concurrents et on voit comment on pourrait se différencier d'eux. »(R6)

"On peut s'appuyer sur des collaborateurs en qui on a confiance pour mettre en cuvre des nouveaux projets, on travaille efficacement avec eux, mais si on travaille avec eux depuis dix ans, ils n'ont plus forcément trop d'imagination, il faut aller chercher les nouvelles idées ailleurs. »(R5)

"En interne on maintient aussi des liens plus ponctuels avec les autres directeurs de sites. [...] C'est parfois très riche, on transfère des idées d'un site à un autre; par exemple je m'inspire actuellement de la division aéronautique pour enrichir l'activité d'un de mes sites sur la logistique des pare-chocs et aller jusqu'à assembler sur le site du client. »(R6)

Les propositions 2 et 4 articulaient caractère dense / lâche du réseau social et capacité d'exploitation / de créativité. Les données collectées sont de nature à illustrer ce groupe de propositions. Les responsables de sites témoignent d'un cœur de réseau social dense, constitué de leurs contacts internes. Autour de ce noyau dense où prédominent les liens forts, les responsables de sites peuvent accéder à des réseaux lâches par le biais de contacts qui agissent pour eux comme des «brokers » d'informations et d'idées nouvelles : ces contacts peuvent ici encore être des interlocuteurs clients ou fournisseurs ou des contacts internes, mais aussi des contacts qui sortent du champ de la socialisation professionnelle, par exemple, des liens familiaux ou amicaux. L'encadré 4 illustre ce propos.

L'analyse comparée des entretiens ne permet finalement pas de mettre en évidence une contingence sur le profil du répondant: il n'y a pas de différence apparente entre les responsables de sites dédiés ou de sites multi-clients quant à la structure et à la nature du réseau ou à son impact sur la performance. En revanche nous faisons l'hypothèse d'une contingence probable liée au positionnement stratégique du prestataire de services logistiques. Nous remarquons en effet que les PSL les plus investis dans la recherche d'une position stratégique forte au sein des supply chains (les grands groupes internationaux offrant des palettes de services logistiques larges et pour certains à forte valeur ajoutée : PSL numérotés ici 1,6 et 8) formalisent des « réseaux d'innovation » par la création de services ingénierie dont la mission est de proposer des innovations aux responsables de sites notamment par une activité de veille. Ceux au contraire qui restent le plus fortement ancrés dans les activités les plus traditionnelles du transport s'insèrent dans des réseaux très denses institutionnalisés, les organisations professionnelles du transport, dont les apports se traduisent clairement plus en termes d'exploitation que d'innovation: comme le résumait un responsable de site à dominante transport, "mes échanges les plus fréquents, quotidiens, c'est en interne avec la $D G$, et avec des collègues au sein des organisations professionnelles comme l'AOTM, l'AFNTR, l'UIM et l'UMF. L'essentiel des échanges porte sur des aspects sociaux et réglementaires, sur l'actualité du secteur. Là on se connaît tous, on cherche à avoir des informations utiles au quotidien, par exemple en ce moment sur les mouvements de grève au PAM [Port Autonome de Marseille]. » 


\section{Encadré 4 - Verbatim : réseau lâche et créativité}

"Les fournisseurs de chariots, racks, quais, ou d'autres équipements sont de bonnes sources d'information car ils voient plus de dossiers que vous, sur des activités très variées : ils peuvent apporter des idées d'innovations techniques dans l'installation des entrepôts. [...] Ce n'est pas nécessairement parce qu'on les connaît bien ou qu'on échange souvent avec eux, cela peut être un commercial qui nous aura démarché une fois et qui aura lancé une bonne idée. » (R1)

"Avec le responsable logistique de [client X], on échange sur ce que font leurs autres prestataires sur l'Europe, il cherche à tirer le meilleur de tous, c'est presque du benchmarking pour nous. Je vois grâce à lui des marges d'évolution et des nouvelles approches des concurrents. » $(R 1)$

"En interne on trouve des sources sur des questions techniques : le service ingénierie est là pour proposer de nouvelles approches, ils sont là pour développer des innovations technologiques ou dans l'organisation. Eux c'est leur métier d'être en veille sur les évolutions, de regarder ce qui se fait ailleurs, même en dehors du secteur de la prestation logistique. »(R6)

"Mon responsable de projet, c'est souvent grâce à lui qu'on propose de nouvelles idées au client. Il est très différent de moi : il a une formation différente et il a une expérience d'un secteur particulier (la logistique du froid pour l'industrie agro-alimentaire) alors que moi j'ai commencé dans le transport de volumineux; et puis aussi il a encore des contacts avec ses anciens collègues et ça lui permet de voir autre chose, du coup il me donne un regard différent et c'est propice à innover dans la façon de faire les choses. » (R7)

"Mon frère est responsable syndical, dans un secteur complètement différent mais il me donne beaucoup d'infos et d'idées pour me sortir parfois de gros problèmes; par exemple il m'a donné une solution pour me débarrasser d'un salarié dangereux à laquelle je n'aurais jamais pensé. »(R8)

\section{Conclusion}

Les entretiens exploratoires conduits nous permettent de confirmer l'intérêt d'une recherche liant réseau social des responsables de sites de prestation logistique et performance générée pour les sites. Les propositions formulées à travers la revue de littérature ont ainsi été confortées et enrichies. Confortées, parce qu'il apparaît en effet que les réseaux denses et/ou constitués de liens forts soutiennent les responsables de sites dans l'exploitation des ressources de leurs sites, et que les réseaux lâches et/ou constitués de liens faibles permettent aux responsables de sites de penser des solutions plus créatives. Enrichies, parce que la description des réseaux sociaux développés par les responsables de sites permet de suggérer une contingence liée au positionnement stratégique des prestataires de services logistiques.

Notons que l'analyse des entretiens a révélé un déséquilibre en faveur des liens forts, nettement plus évoqués par les responsables de sites que leurs liens faibles. Nous pensons que ce déséquilibre s'explique en partie par un biais inhérent à la technique d'entretien utilisée, qui a conduit les responsables de sites à discuter plus spontanément de leurs liens forts. Cependant nous pensons que ce déséquilibre est également révélateur d'un ancrage encore très marqué des responsables de sites dans l'optimisation de l'exploitation des sites.

La méthodologie déployée dans cette recherche n'est pour autant pas exempte de limites. Deux d'entre elles nous semblent pouvoir constituer des pistes de recherche fructueuses. Dans 
le but d'améliorer la validité interne des résultats, il apparaît intéressant d'envisager d'interroger les «interlocuteurs » des responsables de sites rencontrés. Par ailleurs et afin d'améliorer la validité externe des résultats il serait pertinent de mettre en œuvre une méthodologie quantitative permettant de mesurer la portée explicative du réseau social des responsables de sites de prestation logistique sur leur capacité à améliorer la performance de leurs sites, par une meilleure exploitation et par la mise en œuvre de solutions innovantes.

\section{Bibliographie}

C. Argyris et D. Schön (1978), Organizational Learning: A Theory of Action Perspective, Addison Wesley, Reading, MA.

B. Ashenbaum, A. Maltz et E. Rabinovich (2005), «Studies of trends in third-party logistics usage: what can we conclude? », Transportation Journal, Vol. 44, No. 3, p. 39-50.

M. Berglund, P. Van Laarhoven, G. Sharman et S. Wandel (1999), « Third-party logistics: is there a future? », The International Journal of Logistics Management, Vol. 10, No. 1, p. 59-70.

F. Brulhart (2005), «Proximité et réussite des partenariats verticaux logistiques dans le secteur agroalimentaire français », Management International, Vol. 9, No. 4, p. 17-30.

F. Brulhart et S. Claye-Puaux (2008), «Le réseau social, clé de l'agilité et de l'innovation des supply chains ? », in: M. Lecoutre et $\mathrm{P}$. Lièvre, Management et réseaux sociaux : ressource pour l'action ou outil de gestion?, Hermès, Paris.

R. Burt (1992), Structural holes: the social structure of competition, Harvard University Press, Cambridge, MA.

C. Camman et L. Livolsi (2007), «Enjeux et difficultés de la gestion des cadres intermédiaires chez les prestataires de services logistiques », Logistique \& Management, Vol.15, No. 2, p. 43-54.

M. Christopher (2000), «The agile supply chain: competing in volatile markets », Industrial Marketing Management, Vol. 29, No. 1, p. 37-44.

S.A. Chung, H. Singh, et K. Lee (2000), «Complementarity, Status Similarity and Social Capital as Drivers of Alliance Formation », Strategic Management Journal, Vol. 21, No. 1, p. 1-21.

JS. Coleman (1988), « Social capital in the creation of human capital », American Journal of Sociology, Special Issue, Vol. 97, p. 95-120.

JS. Coleman (1990), Foundations of Social Theory, Harvard University Press, Cambridge, MA.

M. Crozier et E. Friedberg (1977), L'acteur et le système, Editions du Seuil, Paris.

J. Dyer et W. Chu (2003), «The role of trustworthiness in reducing transaction costs and improving performance: empirical evidence from the United States, Japan and Korea », Organization Science, Vol. 14, p. 57-68.

N. Fabbe-Costes (2005), «La gestion dynamique des supply chains des entreprises virtuelles », Revue Française de Gestion, No. 156, p. 151-166.

S. Floyd et P. Lane (2000), «Strategizing throughout the organization: managing role conflict in strategic renewal », Academy of Management Review, Vol. 25, No. 1, p. 154-177.

F. Fulconis et G. Paché (2005), «Piloter des entreprises virtuelles, quel rôle pour les prestataires de services logistiques ? », Revue Française de Gestion, No. 156, p. 167186.

M. Granovetter (1973), «The Strength of Weak Ties », American Journal of Sociology, Vol. 78, No. 6, p. 1360-1380.

M. Granovetter (1983), « The strength of weak ties: a network theory revisited », Sociological Theory, Vol. 1, p. 201-233. 
M. Granovetter (1992), «Problems of explanation in economic sociology », in: N. Nohria, R.G. Eccles (Eds), Networks and organizations: structure, form and actions, Harvard Business School Press, Boston, MA, p. 25-36

S. Hertz et M. Alfredsson (2003), « Strategic development of third-party logistics providers », Industrial Marketing Management, Vol. 32, p. 139-149.

Q.N. Huy (2001), «In praise of middle managers », Harvard Business Review, Vol. 79, No. 8, p. $72-80$.

M. Ingham et C. Mothe (2003), «Apprentissage et Confiance au sein d'une Alliance Technologique », XIIième Conférence Internationale de l'Association Internationale de Management Stratégique, 4,5 et 6 juin, Tunis.

P. Kale, J.H. Dyer et H. Singh (2002), «Alliance Capability, Stock Market Response, and Long Term Alliance Success: The Role of the Alliance Function », Strategic Management Journal, Vol. 23, No. 8, p. 747-768.

A.M. Knemeyer et P.R. Murphy (2005), «Exploring the potential impact of relationship characteristics and customer attributes on the outcomes of 3PL arrangements », Transportation Journal, Vol. 12, No. 2, p. 5-19.

D.R. Krause, R.B. Handfield et B.B. Tyler (2007), « The relationships between supplier development, commitment, social capital accumulation and performance improvement », Journal of Operations Management, Vol. 25, p. 528-545.

K.-H. Lai (2004), «Service capability and performance of logistics service providers », Transportation Research Part E, Vol. 40, p. 385-399.

D.A. Levinthal et M. Fichman (1988), «Dynamics in interorganizational attachments: auditor-client relationships », Administration Science Quaterly, Vol. 33, p. 345-369.

R. Lieb et K. Butner (2007), «The North American third party logistics industry in 2006: the provider CEO perspective », Transportation Journal, Vol. 15, No. 1, p. 40-52.

A. Marasco (2008), «Third-party logistics: a literature review», International Journal of Production Economics, Vol. 113, p. 127-147.

B. McEvily et A. Marcus (2005), «Embedded ties and the acquisition of competitive capabilities », Strategic Management Journal, Vol. 26, p. 1033-1055.

M.B. Miles et A.M. Huberman (1994), Qualitative data analysis, 2nd edition, Sage Publications, London.

J. Mohr et R. Spekman (1994), «Characteristics of Partnership Success: Partnership Attributes, Communication Behavior and Conflict Resolution Techniques », Strategic Management Journal, Vol. 15, p. 165-152.

P. Moran (2005), «Structural vs. relational embeddedness: social capital and managerial performance », Strategic Management Journal, Vol. 26, No. 12, p. 1129-1151.

J. Nahapiet et S. Goshal (1998), «Social capital, intellectual capital and the organizational advantage », Academy of Management Review, Vol. 38, No. 2, p. 242-266.

J.E. Perry-Smith (2006), «Social, yet creative: the role of social relationships in facilitating individual creativity », Academy of Management Journal, Vol. 49, No. 1, p. 85-101.

JM. Podolny (2001), « Networks as the pipes and prisms of the market », American Journal of Sociology, Vol. 107, No. 1, p. 33-60.

JM. Podolny et J.N. Baron (1997), «Resources and relationships: social networks and mobility in the workplace », American Sociological Review, Vol. 65, No. 2, p. 673-693.

T. Roques et M. Michrafy (2003), «La prestation de services logistiques en France en 2002 : perception des acteurs et évolution des pratiques », Logistique \& Management, Vol. 11 No. 2, p. 7-26.

T. Sauvage (2003), «The relationship between technology and third-party logistics providers », International Journal of Physical Distribution and Logistics Management, Vol. 33, No. 3, p. 237-253. 
T. Sauvage (2004), «Quelles relations contractuelles pour l'externalisation logistique ?», Revue d'Economie Industrielle, Vol. 106, p. 26-42. 